\section{Protonenresonanz-Untersuchungen über die konstellative Beweglichkeit im substituierten 1.2-Dithianring ${ }^{1}$}

Von Arthur Lütrringhaus und Siegrried Kabuss

Chemisches Laboratorium der Universität Freiburg/Brsg.,

Wilhelm Maier und Horst Friebolin

Institut für Elektrowerkstoffe Freiburg/Brsg.

(Z. Naturforschg. 16 b, 761—762 [1961] ; eingeg. am 12. August 1961)

Die Disulfidgruppierung besitzt im energetischen Minimum diedrische Anordnung mit den beiden $\mathrm{C}-\mathrm{S}$-Bindungen in senkrecht zueinander orientierten Ebenen. Infolge dieser Struktur liegen organische Disulfide $\mathrm{R}-\mathrm{S}-\mathrm{S}-\mathrm{R}$ in zwei enantiomeren Konstellationen vor. Das Behinderungspotential für die Umwandlung der beiden Antipoden ineinander beträgt bei offenkettigen Disulfiden mindestens $10 \mathrm{kcal} / \mathrm{Mol}{ }^{2}$.

Im Rahmen von Untersuchungen über die entsprechende Molekularasymmetrie cyclischer Disulfide ${ }^{1}$ haben wir eine Reihe substituierter 1.2-Dithiane synthetisiert und mit Hilfe der Protonenresonanz-Spektren die Aktivierungsenergie für die Umwandlung der Enantiomeren bestimmt.

Für die Messungen fanden wir vicinal cis (e, a) disubstituierte Systeme geeignet, z. B. 4.5-cis-Diacetoxydithian-(1.2) (III). Die beiden Konformeren sind Antipoden, auch in bezug auf die beiden cis-Substituenten. Die Darstellung von III erfolgte durch dehydrierenden Ringschluß aus meso-1.4-Dimercapto-butandiol(2.3) ${ }^{3}$ (I) zu (II) und anschließende Acetylierung mit Acetanhydrid/Pyridin ${ }^{4}$.
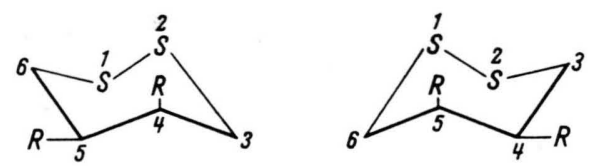

III

Die entsprechenden trans (e.e)-Verbindungen konnten auf demselben Wege aus IV dargestellt werden ${ }^{4,5}$.

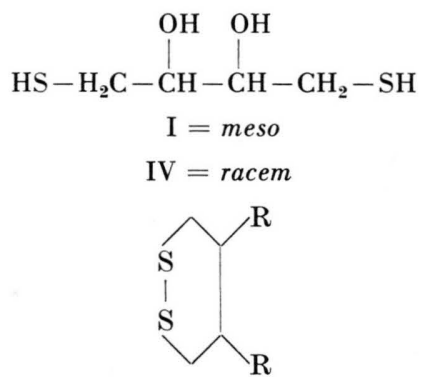

1 Cyclische Disulfide, IV.; III. Mitt.: A. Lütrringhaus u. A. B Rechlin, Chem. Ber. 92, 2271 [1959].

2 D. W. Scott, H. L. Finke, J. P. Cullough, M. E. Gross, R. E. Pennington u. G. Wedpington, J. Amer. chem. Soc. 74, 2478 [1952] ; D. W. Scott, H. L. Finke, M. E. Gross, W. R. Guthrie u. H. M. Hufrmann, J. Amer. chem. Soc. 72, 2424 [1950].

3 R. M. Evans, J. B. Fraser u. L. N. Owen, J. chem. Soc. [London] 1949, 248.

$$
\begin{aligned}
& \text { II } R=\mathrm{OH} \text { cis } \quad \text { Schmp. }=128-129^{\circ}, \lambda_{\max }=289 \mathrm{~m} \text {, } \\
& \text { III } \mathrm{R}=\mathrm{O} \cdot \mathrm{CO} \cdot \mathrm{CH}_{3} \text { cis } \\
& \text { Schmp. }=74-75^{\circ}, \lambda_{\max }=287 \mathrm{~m}, \\
& \mathrm{~V} \quad \mathrm{R}=\mathrm{OH} \text { trans } \text { Schmp. }=129-130^{\circ}, \lambda_{\max }=286 \mathrm{~m} \text {, } \\
& \text { VI } \quad \mathrm{R}=\mathrm{O} \cdot \mathrm{CO} \cdot \mathrm{CH}_{3} \text { trans } \\
& \text { Schmp. }=54-55^{\circ}, \lambda_{\max }=283 \mathrm{~m} .
\end{aligned}
$$

Die Aktivierungsenergie für die Umwandlung der beiden Sesselformen ineinander wurde bei III bestimmt. Das komplizierte Spektrum der Ringprotonen ist hierfür nicht geeignet. Die Protonen der Acetylgruppe dagegen zeigen bei Zimmertemperatur eine einzelne scharfe Absorptionsbande, die infolge der unterschiedlichen chemischen Verschiebung (,chemical shift“) der Protonen von axialem und äquatorialem Acetyl bei Temperaturen unterhalb $-8{ }^{\circ} \mathrm{C}$ in zwei Linien $\left(\Delta v_{\infty}=9,3 \mathrm{~Hz}\right.$ bei $\left.-60{ }^{\circ} \mathrm{C}\right)$ aufspaltet. Aus der Temperaturabhängigkeit der Aufspaltung ermittelten wir nach dem Verfahren von Gutowsky ${ }^{6}$ die Aktivierungsenergie zu $\Delta \mathrm{H}^{+}=12,0 \mathrm{kcal} / \mathrm{Mol}^{7}$. Mit Hilfe der E y ring - Gleichung berechneten wir die freie Aktivierungsenthalpie zu $\Delta \mathrm{G}^{\ddagger}=13,9 \mathrm{kcal} / \mathrm{Mol}$.

An dem von uns etwa gleichzeitig ${ }^{4}$ mit Clateson, Androes und $\mathrm{Calvin}^{8}$ auf etwas anderem Wege synthetisierten 4.4.5.5-Tetradeuterodithian (VII) bestimmten wir das Behinderungspotential für den unsubstituierten Dithianring zu $\Delta \mathrm{H}^{\mp}=11,5 \mathrm{kcal} / \operatorname{Mol}\left(\Delta v_{\infty}=12,3 \mathrm{~Hz}\right.$ bei $-70{ }^{\circ} \mathrm{C}$, Aufspaltungstemperatur: $\left.-48{ }^{\circ} \mathrm{C}\right)^{7}$. Die Fehler sind hier jedoch größer als im Falle von III, da die Spin-Spinkopplung eine Aufspaltung zu einem ABQuartett hervorruft und zusätzliche Kopplung mit den Deuteronen vorliegt. Für die freie Aktivierungsenthalpie $\Delta \mathrm{G} \neq$ erhielten wir den Wert von $11,7 \mathrm{kcal} / \mathrm{Mol}$ in guter Ubereinstimmung mit den oben genannten Autoren.

Die maximalen Aufspaltungen $\Delta v_{\infty}$ der Acetylprotonensignale bei III und der Ringprotonensignale bei VII sind nur wenig voneinander verschieden. Dadurch ergibt sich für die Lebensdauer $\tau$ der enantiomeren Formen bei den Temperaturen von $-8{ }^{\circ} \mathrm{C}$ (III) und $-48{ }^{\circ} \mathrm{C}$ (VII) jeweils etwa derselbe Wert von

$$
\tau=\sqrt{2 / \pi} \Delta v \approx 1 / 20 \mathrm{sec} .
$$

Bei gleicher Temperatur ist also die Beweglichkeit durch die Substitution merklich herabgesetzt. Die Abhängigkeit der Aktivierungsenergie und -entropie von Größe und Art der Substituenten wird z. Z. noch untersucht.

${ }^{4}$ Aus der Dissertation von S. Kabuss, Freiburg/Br. 1961.

5 Diplomarbeit S. Kabuss, Freiburg 1959.

${ }^{6}$ H. S. Gutowsky u. C. H. Holm, J. chem. Physics 25, 1228 [1956].

7 Teil der Dissertation von H. Friebolin, Freiburg.

8 G. Clateson, G. M. Androes u. M. Calvin, J. Amer. chem. Soc. 82, 4428 [1960] ; Die Autoren berechneten mittels der E y r in g schen Gleichung die freie Aktivierungsenthalpie $\mathrm{G}^{\neq}$zu $11,6 \mathrm{kcal} / \mathrm{Mol}$. 
Die Einführung einer Doppelbindung in den 6-gliedrigen Disulfid-Ring macht das System durch Verflachung beweglicher: Sowohl 2.3-Dithiatetralin (VIII) ${ }^{9}$ als auch 1.2-Dithiacyclohexen-(4) (IX) zeigen bei $-80{ }^{\circ} \mathrm{C}$ keine Anzeichen von Aufspaltung durch chemische Verschiebung. Aus der Linienverbreiterung unterhalb $-90{ }^{\circ} \mathrm{C}$ konnte für das 2.3-Dithiatetralin (VIII) die freie Aktivierungsenthalpie $\Delta \mathrm{G}^{ \pm}$zu $8,5 \mathrm{kcal} / \mathrm{Mol}$ abgeschätzt werden.

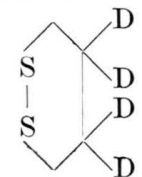

VII

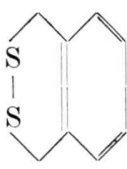

VIII

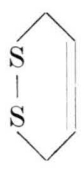

IX
Die Darstellung von IX ${ }^{\mathbf{1 0}}$, das von uns erstmalig in reiner Form (Schmp. $20{ }^{\circ} \mathrm{C}, \lambda_{\max }=283 \mathrm{~m} \mu$ ) gewonnen werden konnte, erfolgte durch Dehydrierung von cis1.4-Dimercaptobuten-(2) (X) in 70-proz. Ausbeute ${ }^{4,5}$. $\mathrm{X}$ erhielten wir über das Diacetat (XI) aus cis-1.4-Dichlorbuten-(2) ${ }^{11}$ durch Umsetzung mit K-Thiolacetat und Na-Methylat-katalysierte Umesterung mit Methanol. Reines trans-1.4-Dimercaptobuten-(2) (XIII) ließ sich analog über das kristallisierte Diacetat (XIV) aus festem trans-1.4-Dibrombuten-(2) leicht darstellen. Die beiden isomeren Mercaptane wurden durch ihre Bis- $p$ nitrobenzoate charakterisiert (XII und XV).

$$
\begin{aligned}
& \mathrm{RS}-\mathrm{H}_{2} \mathrm{C}-\mathrm{CH}=\mathrm{CH}-\mathrm{CH}_{2}-\mathrm{SR} \\
& \mathrm{X} \quad \mathrm{R}=\mathrm{H} \text { cis } \quad \mathrm{Sdp} . \quad=80-81^{\circ} / 11 \mathrm{~mm} \\
& \mathrm{XI} \quad \mathrm{R}=\mathrm{CO} \cdot \mathrm{CH}_{3} \text { cis } \quad \mathrm{Sdp} . \quad=81-83^{\circ} / 0,1 \mathrm{~mm} \\
& \text { XII } \mathrm{R}=\mathrm{CO}-\longrightarrow-\mathrm{NO}_{2} \text { cis } \quad \text { Schmp. }=158-159^{\circ}
\end{aligned}
$$

9 A. Lütrtinghaus u. K. Hägele, Angew. Chem. 67, 304 [1955].

10 A. Schöberl u. H. GräfJe, Liebigs Ann. Chem. 614, 66 [1958].

$$
\begin{aligned}
& \text { XIII } \mathrm{R}=\mathrm{H} \text { trans } \quad \text { Sdp. }=81-82^{\circ} / 11 \mathrm{~mm} \\
& \mathrm{XIV} \quad \mathrm{R}=\mathrm{CO} \cdot \mathrm{CH}_{3} \text { trans } \quad \text { Schmp. }=44-45^{\circ} \\
& \mathrm{XV} \quad \mathrm{R}=\mathrm{CO}-\longrightarrow-\mathrm{NO}_{2} \text { trans } \mathrm{Schmp} .=182-183^{\circ}
\end{aligned}
$$

Ein cis-ankondensierter Fünfring führt ebenfalls nicht zu einer Versteifung des Dithianringes, wie sich am Beispiel des Aceton-acetals (XVI) von II (Schmp. $\left.\left.=48-49^{\circ} \mathrm{C}\right), \lambda_{\max }=284 \mathrm{~m} \mu\right)$ zeigen ließ. Der Dithianring in XVI

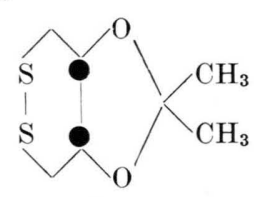

XVI

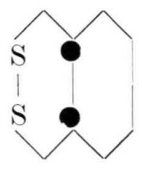

XVII ist noch bei $-70{ }^{\circ} \mathrm{C}$ beweglich. Das Kernresonanzspektrum der Ring-Protonen entspricht bei tiefen Temperaturen annähernd dem des Diacetats III bei Zimmertemperatur.

Die cis-Angliederung eines hydrierten Sechsringes, z. B. im cis-2.3-Dithiadekalin (XVII) ${ }^{12}$, setzt die Beweglichkeit des Dithianringes stark herab. Das System zeigt bereits bei Zimmertemperatur breite, unaufgelöste Banden, die unterhalb $0^{\circ} \mathrm{C}$ zu einem Linienspektrum aufspalten. Die freie Aktivierungsenthalpie $\triangle \mathrm{G} *$ ließ sich zu 15,5 kcal/Mol abschätzen. Zur exakten Bestimmung des $\Delta \mathrm{G}^{\neq}$und der Aktivierungsenergie $\Delta \mathrm{H}^{\neq}$soll das Spektrum durch Deuterierung vereinfacht werden.

Die Kernresonanzspektren wurden mit dem Varian-Spektrometer V 4302 $(60 \mathrm{MHz})$ des Instituts für Elektrowerkstoffe aufgenommen. Wir danken Herrn Professor Dr. R. Mecke für die Uberlassung des Geräts.

11 Darstellung nach R. Huisgen u. E. Laschtuvka, Chem. Ber. 93, 65 [1960].

12 A. Brechlin, Dissertation Freiburg/Br. 1956.

\section{Thia-Aromaten $\mathrm{V}^{1}$ 9-Thia-phenanthrenium-perchlorat}

\section{Von Arthur Lüttringhaus und Albrecht Kolb}

Chemisches Laboratorium der Universität Freiburg i. Br. (Z. Naturforschg. 16 b, 762-763 [1961] ; eingeg. am 12. August 1961)

Von den unsubstituierten Grundkörpern der ThiaAromaten wurden bisher dargestellt das 9-Thia-Analoge des Anthracens, das Thioxanthyliumchlorid ${ }^{2}$, die zweikernigen 1- und 2-Thia-naphthalinium-Salze ${ }^{3,4}$ und kürzlich auch das einkernige Thia-Analoge des Benzols

1 IV. Mitt.: N. Engelhard u. A. Kolb, Angew. Chem. 73, 218 [1961].

2 S. Smiles u. Th. Hilditch, J. chem. Soc. [London] 99, 145 [1910].

3 A. Lütrringhaus u. N. Engelhard, Naturwissenschaften 44, 584 [1957]; Chem. Ber. 93, 1525 [1960]. in Gestalt unsubstituierter Thiopyrylium-Salze mit verschiedenen Anionen 5, ${ }^{6}$. Wir konnten diese Reihe jetzt abschließen durch Darstellung des 9-Thia-phenanthrenium-perchlorates (III) auf drei verschiedenen Wegen.

a) o-Nitrobenzylbromid wird mit Na-thiophenolat zum Phenyl-(2-nitrobenzyl)-sulfid (aus Alkohol Schmp. $64^{\circ}$ ) umgesetzt, dieses mit Eisen in Eisessig-Toluol oder in besonders glatter Reaktion mit R a n e y - Nickel und Wasserstoff (Normaldruck, 25 ${ }^{\circ}$ ) in Methanol zum Phenyl-(2-aminobenzyl)-sulfid (I) (aus Petroläther Schmp. $81^{\circ}$, Benzoylderivat Schmp. $172^{\circ}$ ) reduziert. I wird über die Diazoniumverbindung durch P s c h or r -

4 W. Bonthrone u. D. H. Reid, Chem. and Ind. 1960, 1192.

5 R. Petrit, Tetrahedron Letters 1960, Nr. 23, 11 [1960].

6 A. Lütringhaus u. N. Engelhard, Angew. Chem. 73, 218 [1961]. 